Check for updates

Cite this: RSC Adv., 2017, 7, 31691

Received 2nd May 2017

Accepted 12th June 2017

DOI: 10.1039/c7ra04912a

rsc.li/rsc-advances

\section{One-minute self-assembly of millimetre-long DAST crystalline microbelts via substrate-supported rapid evaporation crystallization $\uparrow$}

\author{
Tian Tian, (D) ${ }^{a}$ Bin Cai, ${ }^{\star a}$ Tianming Ye, ${ }^{a}$ QingQing Cheng, ${ }^{a}$ Peng Zhan, ${ }^{a}$ Gongjie $X u{ }^{a}$ \\ Ling Zhang ${ }^{a}$ and Okihiro Sugihara ${ }^{b}$
}

In this work, we propose a substrate-supported rapid evaporation crystallization (SSREC) method to rapidly self-assemble microbelts of $4-N, N$-dimethylamino- $4^{\prime}-N^{\prime}$-methyl-stilbazolium tosylate (DAST), a benchmark organic nonlinear optical (NLO) crystal. The DAST microbelts formation depends very largely on substrate property and surfactant, and millimetre-long microbelts can be fabricated in less than one minute. The self-assembly driving forces are presumed to be the coulombic interactions which originate from DAST's ionic molecular structure and the large dipole moment of DAST nanocrystals. The DAST microbelt has a second harmonic generation active crystal structure and exhibits featured absorption and fluorescent properties. We believe that all these findings will facilitate applications of DAST in a very broad range of areas such as integrated light modulation, lasing etc. Furthermore, the simplicity of the SSREC may open new avenues in the area of organic NLO one-dimensional materials fabrication.

\section{Introduction}

One-dimensional (1D) self-assembly of organic functional materials into well-defined micro/nanowires or belts has gained increasing interest because of their unique properties for use in electronic, optoelectronic, and photonic nano-devices. ${ }^{1-5}$ Many interactions can possibly be utilized for 1D self-assembly, such as coordination bonding, aromatic $\pi-\pi$ stacking, hydrogen bonding, van der Waals forces, and electrostatic forces. ${ }^{6-10}$ Many different organic materials and methods have been employed to synthesize 1D single-crystal micro/nanostructures. ${ }^{\mathbf{1 1 - 1 9}}$ Organic nonlinear optical (NLO) materials are superior to inorganics in both the speed of their optical response and the magnitude of their NLO properties. ${ }^{20}$ Therefore, organic NLO materials have been intensively researched for applications in frequency conversion, optical parametric oscillation, electro-optic modulation, integrated photonic devices, and terahertz (THz) applications. $^{21} \quad 4$ - $N, N$-Dimethylamino- $4^{\prime}-N^{\prime}$-methyl-stilbazolium tosylate (DAST), an organic ionic crystal recognized as a benchmark organic NLO material, has high electro-optic coefficients $\left(n^{3} r_{11}=455 \pm 80 \mathrm{pm} \mathrm{V}^{-1}\right.$ at $\left.1535 \mathrm{~nm}\right)$, high NLO susceptibility

${ }^{a}$ Shanghai Key Lab of Modern Optical System and Engineering Research Center of Optical Instrument and System, Ministry of Education, University of Shanghai for Science and Technology, Shanghai 200093, China.E-mail: bullcai@gmail.com ${ }^{b}$ Graduate School of Engineering, Utsunomiya University, Utsunomiya 321-8585, Japan

$\dagger$ Electronic supplementary information (ESI) available. See DOI: 10.1039/c7ra04912a $\chi_{111}^{(2)}(-2 \omega, \omega, \omega)=580 \pm 30 \mathrm{pm} \mathrm{V}^{-1}$ at $1535 \mathrm{~nm}$, as well as a low dielectric constant $(5.2,103-105 \mathrm{kHz}){ }^{22,23} \mathrm{THz}$ generation and various electro-optical devices, such as frequency modulators, have been investigated using such materials in recent years. ${ }^{24,25}$ In order to effectively utilize the NLO properties of DAST crystals, an optical waveguide structure is preferred. Many efforts have been devoted to fabricating DAST waveguides such as reactive ion etching, photobleaching and direct electron beam writing. ${ }^{26,27}$ However, obtaining an optical waveguide from bulk crystals remains a significant challenge.

In our previous work, we developed a substrate-supported rapid evaporation crystallization method (SSREC) which can be used for DAST nanocrystals (NCs) fabrication. ${ }^{28}$ In this method, varying the evaporation temperature can effectively control the size of DAST NCs; single nanometre NCs can be easily obtained. On the other hand, NC surfactants can greatly affect the morphologies of organic molecular aggregation. ${ }^{29-31}$ For instance, Zhang and co-workers effectively tuned the morphology of 9,10-diphenylanthracene aggregations using different surfactants via a solution based reprecipitation method, with shapes ranging from an amorphous nano-sphere to crystalline octahedral nanoparticles, crystalline nanowires, and microrods. ${ }^{29}$ Moreover, Huang et al. successfully selfassemble small organic molecules into ultralong microbelts (MBs) in the presence of PEG- $b$-PEI polymer as crystal growth modifier. ${ }^{31}$ In this work, we extend the SSREC method to fabricating 1D DAST single crystal MBs using a polymer-type surfactant. The self-assembly driving forces are presumed to be the coulombic interactions which originate from DAST's 
ionic molecular structure and the large dipole moment of DAST NCs. In contrast to other weak interactions, which require quite a long time under a metastable or quasi-equilibrium condition for the assembly process, coulombic forces act quickly and efficiently, forming millimetre-scale DAST single crystal MBs within one minute.

\section{Experimental}

The DAST powder (Daiichi Pure Chem. Co. Ltd.) and other chemical reagents were used directly without further purification. In order to assemble DAST MBs, glass substrates were treated with octadecyldichlorosilane (OTS), followed by a deep UV radiation treatment to remove the OTS using a Deep UV ozone cleaner (Novascan PSD-UV) with primary output wavelengths of $254 \mathrm{~nm}(90 \%)$ and $185 \mathrm{~nm}(10 \%)$ for $20 \mathrm{~min}$. Water droplet contact angles were $c a .100^{\circ}$ and $20^{\circ}$ before and after removing the OTS, respectively. The hydrophilic glass substrate was then placed on a hot plate and heated to $c a .150{ }^{\circ} \mathrm{C}$. Next, $0.25 \mathrm{mM}$ DAST methanol solution was mixed with a polymertype surfactant (Disperbyk®-111, BYK Additives \& Instruments, acid value: 129) (weight ratio of DAST to surfactant is $1: 1.5$ ), and dropped onto the heated glass substrate. As the methanol rapidly evaporated, the DAST MBs formed immediately. The morphology of the DAST MBs was studied by laser confocal microscopy (Olympus OLS4000-SAT), scanning electron microscopy (SEM, FEI Quanta 200 FEG and Helios Nanolab 600i), and transmission electron microscopy (TEM, JEM 2011). The crystal structure of the MBs was studied by X-ray diffraction (XRD, PuxiXD-3). The absorption and fluorescence properties were investigated by ultraviolet-visible spectroscopy (UV-vis, Shimadzu UV-3100PC) and fluorescent microscopy (Zeiss, Axios), respectively.

\section{Results and discussion}

Fig. 1(a) shows the SEM image of the DAST NCs fabricated at $110^{\circ} \mathrm{C}$ with $50 \mathrm{wt} \%$ cetyl trimethylammonium bromide (CTAB), a low molecular weight surfactant. Due to the CTAB surface modification, the DAST crystals randomly precipitated on the substrate, and the size of the NCs is on the scale of tens of nanometres. Fig. 1(b) is a TEM image of the NCs prepared at $145{ }^{\circ} \mathrm{C}$. At this temperature, the size of the DAST crystals decreased to the single nanometre scale, but no fibrils can be observed. A detailed study on DAST NC fabrication was reported in our previous paper. ${ }^{28}$ Surfactants can dramatically change the morphology of DAST crystals. However, when we changed the CTAB to a polymer-type surfactant, instead of forming randomly precipitated NCs, DAST tended to self-assemble into a fibril structure. Fig. 2(c) shows the DAST fibrils fabricated at $150{ }^{\circ} \mathrm{C}$ using 150 wt\% Disperbyk-111 surfactant (a copolymer with acidic groups with an acid number of 129). The high acid number surfactant can not only modify the DAST NC surface and minimize the NCs' surface energy but can also enrich the DAST NCs precipitating along the copolymer backbone. This helps form the linear seed of the DAST fibrils, therefore playing an extremely important role for the crystalline fibril formation.

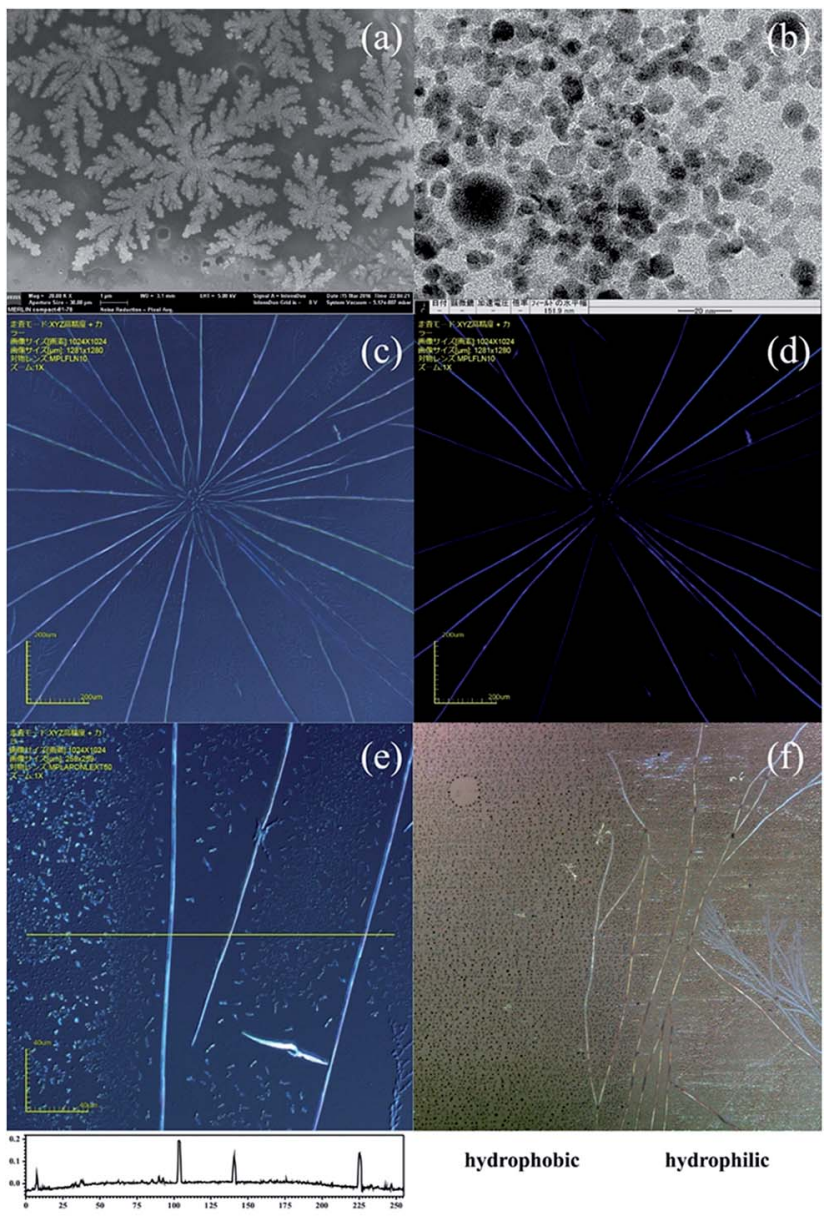

Fig. 1 (a) SEM image of DAST NCs prepared at $110^{\circ} \mathrm{C}$; (b) TEM image of DAST NCs prepared at $145^{\circ} \mathrm{C}$ (c) confocal microscopy image of ultra-long DAST single crystalline MBs prepared by the SSREC method; (d) confocal microscopy image of the DAST MBs under crossed polarization; (e) a zoomed-in image and a cross-sectional height profile showing the belt morphology and uniform size of the DAST MBs; (f) a comparison of DAST crystal growth on surfaces with different properties, left side: hydrophobic, right side: hydrophilic.

Low acid number surfactant was also tried in the experiments. It can also form MBs by similar processes, however, they were much shorter than that of Disperbyk-111 (see ESI $\dagger$ ). Laser confocal microscopy confirms several crystalline fibrils with a radial-like alignment. This radially growing behaviour is very similar to the self-assembly of nanobelts from the central seeding particulate aggregates reported by Zang et al. ${ }^{32}$ However, in our case, no obvious seed can be observed at the centre of the radial pattern. Furthermore, our DAST crystal growth exhibits a much stronger dynamic crystallization property than the reported perylene material. In that reference, to ensure the fibril length and growth direction, the self-assembly process must be under quasi-equilibrium conditions, and consequently, a comparatively long time (typically over 5 days) is required. In our case, however, the self-assembly process is very fast, and typically, DAST crystalline fibrils can form in less than 1 minute (i.e., the time required for the for the methanol solvent to evaporate). This means that a comparably strong interaction 


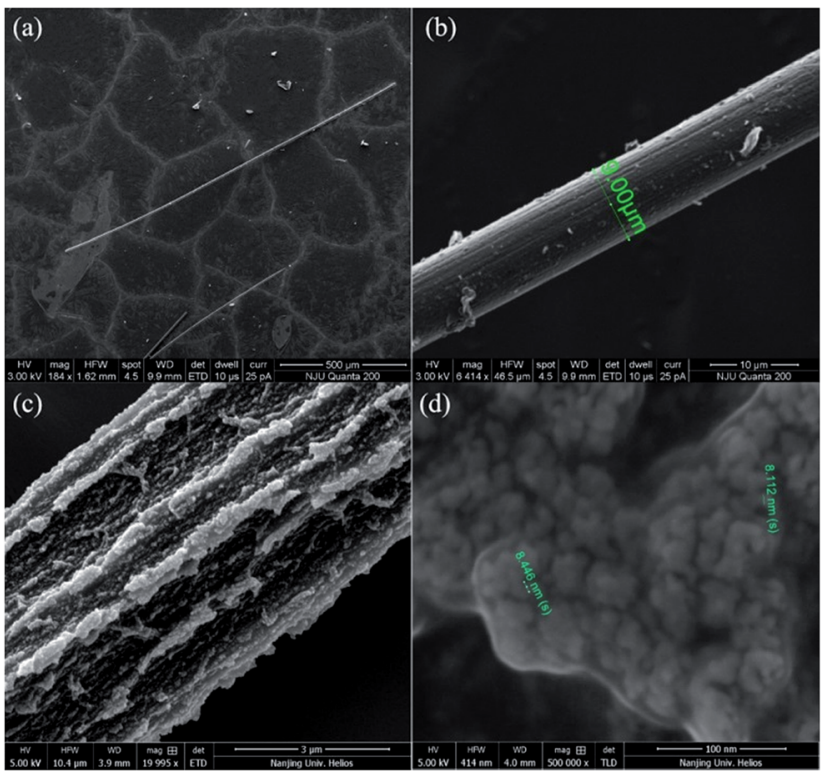

Fig. 2 SEM images of DAST MBs: (a) a DAST MB over $1 \mathrm{~mm}$ in length; (b) a perfectly self-assembled DAST MB with a smooth top surface; (c) an imperfect DAST MB with an unfinished, coarse top surface; (d) a detail of the imperfect DAST MB, exhibiting DAST NC aggregation.

dominates the crystallization. As DAST is an ionic NLO crystal with very strong donor-acceptor $\pi$-conjugation structure, we presume that there are two different coulombic interactions, which dominate the fibril formation. One originates from the electrostatic attraction between the chromophore cations and their counter anions, and the other one is due to the large dipole moment of the DAST NCs. Chiang et al. reported a massive electric dipole moment in the ground state of the DAST sub-microcrystals. ${ }^{33}$ They suggested that for a $10 \mathrm{~nm}$ DAST NC, the dipole moment could be as high as $c a .500 \mathrm{D}$, which benefits their rapid self-assembly. Under these two coulombic interactions, the DAST fibrils can grow to macroscale lengths; most of them are longer than $0.5 \mathrm{~mm}$, and some of them can even reach ca. $3 \mathrm{~mm}$ (see ESI $\dagger$ ). Fig. 1(d) shows microscopic image of DAST crystalline fibrils under crossed polarization. Only when the fibrils were aligned $45^{\circ}$ to the direction of the polarizer, the anisotropy birefringence was maximized (brightest). At a position parallel to the polarizer, the birefringence of the fibrils became minimal (extinguished). This implies that the fabricated DAST fibrils have a single crystalline structure with $\pi-\pi$ stacking molecular alignment throughout the fibril. Fig. 1(e) reveals that the DAST single crystalline fibrils obtained a belt-like morphology. The typical width of the MBs is $1-10 \mu \mathrm{m}$, and the thickness is $0.1-2 \mu \mathrm{m}$, with a width-to-thickness aspect ratio of $c a .5: 1$. In addition to the MBs, the figure also shows many DAST NCs formed on the substrate. The distribution of the NCs is not homogeneous, however, the NC density adjacent to the DAST MBs is obviously lower than the NC density further away. We believe that after the linear seed crystal forms, it absorbs the adjacent DAST solution within the range of $10-20 \mu \mathrm{m}$ by the capillary effect. As the methanol solvent evaporates, the absorbed solution undergoes
DAST NC precipitation and self-assembles due to coulombic forces. Finally, DAST MBs with millimetre-scale can be realized.

In addition to the influence of the surfactant, DAST MB formation also strongly depends on the hydrophilicity of the substrate. Fig. 1(f) shows a comparison of the MB formation on two different surfaces. The left side is hydrophobic due to the OTS surface modification, and the right side is hydrophilic because of the deep UV light irradiation treatment. Clearly, only the hydrophilic surface can form the DAST MBs, indicating there is a very strong surface selectivity.

Fig. 2(a) shows an SEM image of the DAST single crystalline MBs fabricated by the SSREC method. The MB is more than 1 $\mathrm{mm}$ long, and its width is uniform. Fig. 2(b) shows a DAST MB with a well-assembled structure; the $\mathrm{MB}$ has a smooth top surface, and thus, low optical propagation loss can be deduced. However, there are some MBs, which are not well assembled, as shown in Fig. 2(c). In this case, although the MB can still grow to millimetres long, it has an irregular, coarse top surface. We presume that the DAST MB formation undergoes two independent self-assembly processes: linear aggregation and NC reorganization (see ESI $\dagger$ ). The linear aggregation process is obviously faster than the NC reorganization, as demonstrated by the long crystalline MB with an unfinished surface. Fig. 2(d) is a detail of the unfinished surface, where many NCs aggregate randomly on the MB surface. The size of the NCs is approximately $10 \mathrm{~nm}$, which is similar to the crystals formed with the low molecular weight CTAB surfactant.

Fig. 3(a) is the XRD pattern of DAST NCs, Fig. 3(b) is that of DAST MBs and Fig. 3(c) is that of DAST bulk power. ${ }^{34}$ In contrast to the randomly dispersed DAST NCs, the self-assembled DAST MBs only show a diffraction peak indicating the $\{004\}$ face, despite being comprised of numerous DAST NCs. This result indicates that although the DAST NCs are assembled at a very high speed, the strong electrostatic attractions can organize them very well. The peak positions confirm that the DAST MBs have a monoclinic crystal structure, which is a second harmonic

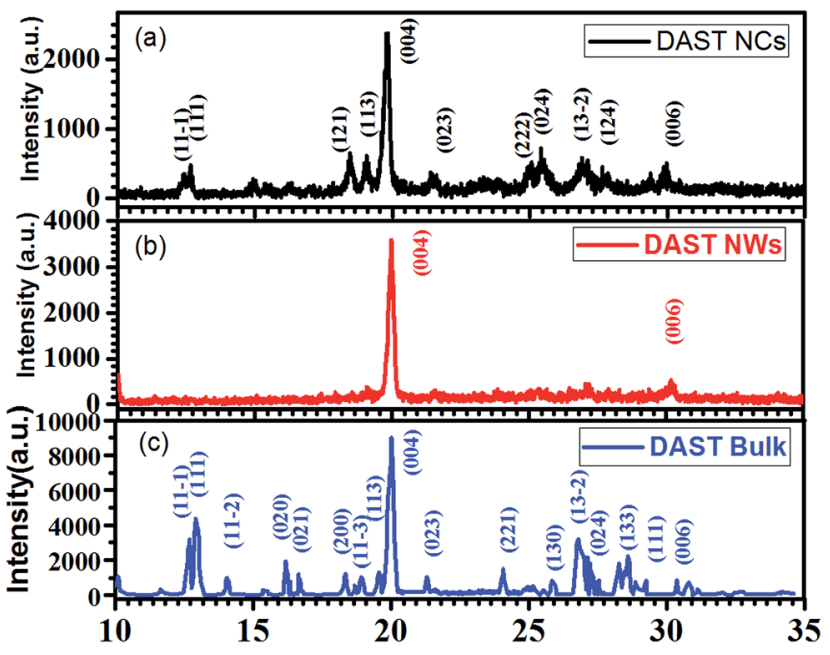

Fig. 3 XRD patterns of (a) DAST NCs, (b) DAST crystalline MBs prepared by the SSREC method and (c) DAST bulk power. ${ }^{34}$ 
generation (SHG) active structure and good for electro-optic or other nonlinear applications. ${ }^{35}$

The UV-vis absorption of the DAST MBs was measured as grown on glass slides, and a typical result is shown in Fig. 4(a) (red line). As references, the absorptions of DAST NCs (blue line) prepared by the SSREC method and a 14.6 $\mu \mathrm{M}$ DAST solution in methanol (black line) are also plotted (all spectra are normalized). Compared to the DAST solution, DAST NCs exhibit a broader absorption band due to hypsochromic and bathochromic shifts (i.e., blue and red shifts, respectively) of the electronic transition of $\pi-\pi$ conjugating system of the $4-N, N$ dimethylamino-4- $N^{\prime}$-methyl-stilbazolium cation. The inset shows a typical diagram of $\mathrm{H}$ - and J-aggregation directions of DAST MBs, the bathochromic shift originates from the Jaggregation of the chromophore cations in a head-to-tail stacking mode, and the hypsochromic shift originates from the $\mathrm{H}$-aggregation in a face-to-face stacking mode. ${ }^{36,37}$ The NCs only exhibit a small expansion in both the blue and red directions, which indicates the size limitation in both aggregating directions. In contrast, the MBs show a small extension in the red direction and a great extension in the blue direction. In other words, due to its $1 \mathrm{D}$ structure, the $\mathrm{H}$-aggregation direction is greatly enhanced.

Fluorescence photography of the DAST MBs is shown in Fig. 4(b). Blue light excites the DAST MBs to emit yellow light, which is very different from the orange fluorescent emission common of the DAST bulk crystals. In other words, the emission expanded toward hypochromatic direction due to the $\mathrm{MB}$ structure. Fig. 4(c) shows the fluorescent spectra of DAST NCs, (a)

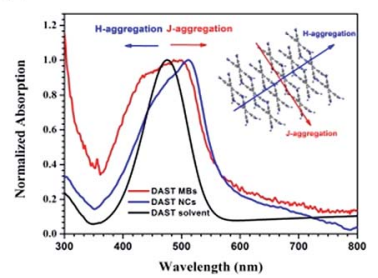

(c)

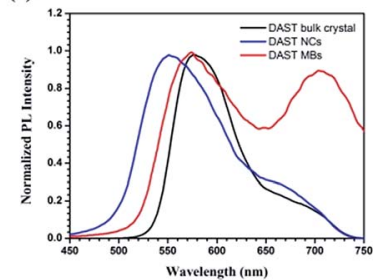

(b)

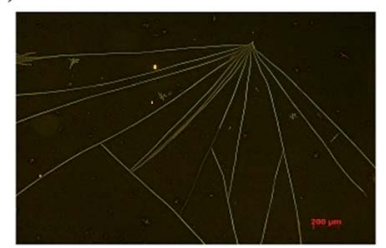

(d)

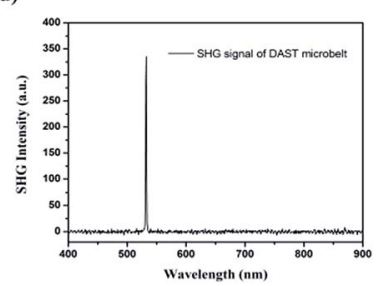

Fig. 4 (a) Normalized UV-vis absorption spectra of DAST MBs (red), NCs (blue) and the methanol solution (black). The DAST MBs have an absorption peak at ca. $498 \mathrm{~nm}$ and a shoulder at ca. $438 \mathrm{~nm}$, while the NC has a absorption peak at ca. $512 \mathrm{~nm}$, and the DAST solution has a peak at $476 \mathrm{~nm}$, the inset is a diagram of $\mathrm{H}$ - and $\mathrm{J}$-aggregation directions of DAST MBs, wherein the tosylate anion groups were removed from the structure for clarity; (b) a fluorescent photograph of DAST MBs excited by a halogen lamp; (c) normalized photo-luminescence spectra of DAST MBs (red), NCs (blue) and bulk crystal (black) excited by a $405 \mathrm{~nm}$ laser; (d) SHG spectrum of DAST MBs excited by a $1064 \mathrm{~nm} \mathrm{cw}$ laser.
MBs and the bulk crystals. Extending the J-aggregation length of chromophores will red-shift the fluorescent spectrum with respect to monomers $;^{37}$ therefore, at the short wavelength side, the DAST bulk crystal has the most bathochromic spectra because its aggregation length is the longest. By contrast, the cut-off wavelength of the DAST NCs spectrum blue-shifts by $c a$. $45 \mathrm{~nm}$, which is due to the NCs' extremely short J-aggregation length. DAST MBs are formed by the aggregation of NCs via self-assembly, and this aggregation will prolong the $\mathrm{J}$ aggregation. Consequently, the DAST MBs exhibit a moderate shift of $c a .20 \mathrm{~nm}$. At the long wavelength side, the DAST MBs exhibit a distinct spectrum expansion with a peak at approximate $720 \mathrm{~nm}$, which is believed to originate from the delocalization of the excited state of molecules due the DAST MB's linear structure. Toward broadband tunable microlaser applications, there are many efforts to tuning the stilbazolium derivatives emission spectra, ${ }^{38}$ obviously our MB structure can offer an excellent solution. A $1064 \mathrm{~nm} \mathrm{cw}$ laser was launched into a DAST MB through a home-made micro-nano fiber and its SHG signal was collected using a microscope (LaicaTCSSP8) and detected by a spectrometer (PG2000-Pro) as shown in Fig. 4(d). We can see that the SHG signal at $532 \mathrm{~nm}$ with FWHM of $1 \mathrm{~nm}$ can be observed. This result proves that the fabricated DAST MBs are SHG active. By consider it XRD data as well, we believe that the MBs have similar NLO ability as that of DAST bulk crystal.

\section{Conclusions}

The SSREC method was successfully extended to self-assemble DAST single crystal MBs. By changing the surfactants, the morphology of DAST crystal can change dramatically. Using a polymer-type surfactant with a high acid value, we were able to obtain ultra-long DAST MBs within a very short time. Our DAST MB has SHG activity crystal structure, expanded fluorescent spectrum and a fine 1D structure, and these properties will facilitate applications of DAST MBs in a very broad area such as integrated light modulation, broadband tunable lasing etc. Although the crystalline MBs quality may need to further improve for practical utilization, we believe the simplicity of the SSREC may make the proposed method highly popular as a new organic NLO materials production technique.

\section{Acknowledgements}

This work was supported by the National Natural Science Foundation of China, Grants No. 61377111, No. 61306118, and NICT International Exchange Program of "Study of Optical Waveguide Device for Terahertz Sensing Using Organic Nanocrystals".

\section{Notes and references}

1 E. W. Meijer and A. P. H. J. Schenning, Nature, 2002, 419, 353.

2 F. J. M. Hoeben, P. Jonkheijm, E. W. Meijer and A. P. H. J. Schenning, Chem. Rev., 2005, 105, 1491. 
3 Y. Yamamoto, T. Fukushima, Y. Suna, N. Ishii, A. Saeki, S. Seki, S. Tagawa, M. Taniguchi, T. Kawai and T. Aida, Science, 2006, 314, 1761.

4 L. Wei, J. N. Yao and H. B. Fu, ACS Nano, 2013, 7, 7573.

5 I. D. Tevis, W. W. Tsai, L. C. Palmer, T. Aytun and S. I. Stupp, ACS Nano, 2012, 6, 2032.

6 X. J. Zhang, J. S. Jie, W. Deng, Q. X. Shang, J. C. Wang, H. Wang, X. F. Chen and X. H. Zhang, Adv. Mater., 2016, 28, 2475.

7 L. Zang, Y. K. Che and J. S. Moore, Acc. Chem. Res., 2008, 41, 1596.

8 M. Koepf, F. Cherioux, J. A. Wytko and J. Weiss, Coord. Chem. Rev., 2012, 256, 2872.

9 L. J. Bu, T. J. Dawson and R. C. Hayward, ACS Nano, 2015, 9, 1878.

10 B. Rybtchinski, ACS Nano, 2011, 5, 6791.

11 K. Balakrishnan, A. Datar, R. Oitker, H. Chen, J. M. Zuo and L. Zang, J. Am. Chem. Soc., 2005, 127, 10496.

12 S. Chen, P. Slattum, C. Y. Wang and L. Zang, Chem. Rev., 2015, 115, 11967.

13 S. Rosenne, E. Grinvald, E. Shirman, L. Neeman, S. Dutta, O. Bar-Elli, R. Ben-Zvi, E. Oksenberg, P. Milko and V. Kalchenko, Nano Lett., 2015, 15, 7232.

14 K. Xiao, J. Tao, Z. W. Pan, A. A. Puretzky, I. N. Ivanov, S. J. Pennycook and D. B. Geohegan, Angew. Chem., Int. Ed., 2007, 46, 2650.

15 A. L. Briseno, S. C. B. Mannsfeld, C. Reese, J. M. Hancock, Y. Xiong, S. A. Jenekhe, Z. Bao and Y. N. Xia, Nano Lett., 2007, 7, 2847.

16 Y. B. Guo, L. Xu, H. B. Liu, Y. J. Li, C. M. Che and Y. L. Li, $A d v$. Mater., 2015, 27, 985.

17 M. He, L. Zhao, J. Wang, W. Han, Y. L. Yang, F. Qiu and Z. Q. Lin, ACS Nano, 2010, 4, 3241.

18 G. M. Sun and C. C. Chu, ACS Nano, 2009, 3, 1176.

19 T. O. Mason, D. Y. Chirgadze and A. Levin, ACS Nano, 2014, 8, 1243.

20 L. R. Dalton, P. A. Sullivan and D. H. Bale, Chem. Rev., 2010, 110, 25.
21 K. S. Rao, A. K. Chaudhary, M. Venkatesh, K. Thirupugalmani and S. Brahadeeswaran, Curr. Appl. Phys., 2016, 16, 777.

22 S. R. Marder, J. W. Perry and W. P. Schaefer, Science, 1989, 245, 626.

23 M. Jazbinsek, L. Mutter and P. Gunter, IEEE J. Sel. Top. Quantum Electron., 2008, 14, 5.

24 I. Katayama, R. Akai, M. Bito, H. Shimosato, K. Miyamoto, H. Ito and M. Ashida, Appl. Phys. Lett., 2010, 97, 021105.

25 L. Mutter, Ph.D. Dissertation, ETH Zurich, Switzerland, 2007.

26 T. Kaino, B. Cai and K. Takayama, Adv. Funct. Mater., 2002, 12, 599.

27 L. Mutter, M. Koechlin, M. Jazbinsek and P. Gunter, Opt. Express, 2007, 15, 16828.

28 T. Tian, B. Cai and O. Sugihara, Nanoscale, 2016, 8, 18882.

29 B. Yang, J. C. Xiao, J. I. Wong, J. Guo, Y. C. Wu, L. Ong, L. L. Lao, F. Boey, H. Zhang, H. Y. Yang and Q. C. Zhang, J. Phys. Chem. C, 2011, 115, 7924.

30 Z. Q. Lin, P. J. Sun, Y. Y. Tay, J. Liang, Y. Liu, N. E. Shi, L. H. Xie, M. D. Yi, Y. Qian and Q. L. Fan, ACS Nano, 2012, 6, 5309.

31 M. Huang, U. Schilde, M. Kumke, M. Antonietti and H. Colfen, J. Am. Chem. Soc., 2010, 132, 3700.

32 Y. K. Che, A. Datar, K. Balakrishnan and L. Zang, J. Am. Chem. Soc., 2007, 129, 7234.

33 H. C. Chiang, T. Iimori, T. Onodera, H. Oikawa and N. Ohta, J. Phys. Chem. C, 2012, 116, 8230.

34 T. Thomasa, J. V. Ramaclus, F. P. Menab, E. Mosquerac, P. Sagayaraja and E. A. Michael, CrystEngComm, 2015, 17, 1989.

35 S. R. Marder, J. W. Perry and C. P. Yakymyshyn, Chem. Mater., 1994, 6, 1137.

36 R. Macchi, E. Cariati, D. Marinotto, D. Roberto, E. Tordin, R. Ugo, R. Bozio, M. Cozzuol, D. Pedron and G. Mattei, J. Mater. Chem., 2010, 20, 1885.

37 A. Miniewicz, K. Palewska, L. Sznitko and J. Lipinski, J. Phys. Chem. A, 2011, 115, 10689.

38 H. Y. Dong, Y. H. Wei, W. Zhang, C. Wei, C. H. Zhang, J. N. Yao and Y. S. Zhao, J. Am. Chem. Soc., 2016, 138, 1118. 